http://jmscr.igmpublication.org/home/ ISSN (e)-2347-176x ISSN (p) 2455-0450 crossref DOI: https://dx.doi.org/10.18535/jmscr/v8i1.58

\title{
Rampant usage of antibiotics in animal bite cases in the era of antibiotic resistance: A cross-sectional study
}

\author{
Authors \\ Swetaleena Ashe*, Tapas Ranjan Behera, Dharitri Mohapatra, Jyotirmayee Panda \\ Sriram Chandra Bhanja Medical College and Hospital, Cuttack, Odisha \\ *Corresponding Author \\ Dr Swetaleena Ashe \\ Department of Community Medicine, SCBMCH, Cuttack, India
}

\begin{abstract}
Background: Wounds inflicted by animal bites are often contaminated by various organisms. Antibiotic resistance is an alarming problem of the present era and judicious use of antibiotics is a must for its prevention. Hence this study was conducted to know the organisms harboring the wounds in animal bite cases and their drug sensitivity pattern.

Objectives: To study the socio-demographic and behavioral factors of patients with wounds caused by animal bites, to enumerate the organisms found in the wound, and to find their drug sensitivity pattern with special reference to age of cases and site of bite.

Methods: Data was collected using pre-designed and pre-tested questionnaire from cases of animal bites with deep/lacerated wounds attending the anti-rabies clinic (ARC) of a tertiary care teaching hospital of Central Odisha from March till September 2019.

Results: A total of 123 cases of animal bites were registered for the study of which 75 cases met the inclusion criteria. Majority (46.7\%) of the cases were geriatrics. Staphylococcus (52\%) was found in majority of the wounds followed by Pseudomonas (19.2\%), Acinetobacter (16.2\%) and Klebsiella (14.7\%). Linezolid (80\%), showed the maximum sensitivity followed by Cefepime (69.2\%), Amoxycillin clavulanic acid (60\%), Tobramycin (50\%) and Cotrimoxazole (49\%). Children $<15$ years were more sensitive to Cotrimoxazole than adults OR 3.7 (1.22-11.51).

Conclusion: Staphylococcus was found to be the major organism harboring the wounds. Geriatrics were found to nearly three times highly sensitive to Linezolid and children were nearly four times more sensitive to Cotrimoxazole. Resistance was marked more among injectable drugs, hence judicious use of antibiotics is the key to prevent antibiotic resistance.

Keywords: Antibiotic resistance, Drug resistance, Rabies, Odisha, Animal bite.
\end{abstract}

\section{Introduction}

Dogs account for $99 \%$ of rabies transmission in humans. ${ }^{1}$ Rabies being a completely preventable disease, vaccination along with supportive treatment should bring the morbidity caused by Rabies to zero. The major wounds of Category III exposure are heavily contaminated with the offending organisms of the oral cavity of the animal. Among the dog's saliva, pathogens such as Staphylococcus, Enterococcus, Pseudomonas, Citrobacter and Klebsiella were mainly found. ${ }^{2}$ 
Infection can lead to a serious complication of sepsis. Hence, proper wound care is of utmost importance as of management of animal bite. In the era of 'Antibiotic Resistance' empirical administration without sensitivity tests of antibiotics would be unethical and hence this study is an attempt to properly know the organisms contaminating such wounds and prescribing drugs sensitive to them. The objectives are to study the socio-demographic and behavioral factors of patients with deep/lacerated wounds caused by animal bites, to enumerate the organisms found in the wound and to find their drug sensitivity pattern with special reference to age of cases and site of bite.

\section{Material and Methods \\ Study Setting}

The snapshot study was carried out in the Anti Rabies Clinic of a tertiary care teaching hospital of Central Odisha from March - September 2019.

\section{Recruitment}

Patients aged more than one year, referred from peripheral health institutions with deep/lacerated wound due to animal bite which was large enough to get a swab and agreed to give consent (parents giving consent for minors) were included in the study. Patients who had taken antimicrobial agents within 72 hours before reporting to the ARC and had minor injuries with bleeding were excluded. All the prescriptions from respective referral hospitals were evaluated for antibiotics prescribed. Purposive sampling was done to select cases of Category III animal bite to obtain an adequate number of cases. Finally during the study period (data collection) over a period of four months a total of 123 such cases could be included for the study.

\section{Methodology}

A face to face interview was carried out using an interviewer administered predesigned, pretested semi-structured questionnaire involving the following data e.g age, gender, residence, day of reporting since the bite, any medications taken, type of animal, type of bite and traceability of the animal. The swab from the wounds of these patients were collected by a sterile swab stick and immediately (within 15 minutes) sent to the Department of Microbiology of the Institution, for Culture and Sensitivity of the wound swab.

Microbiological specimens were collected aseptically using sterile swabs from the edge of the open lacerated wounds and appropriately labelled. These specimens in the Department of Microbiology were then swabbed onto Blood agar and Mac Conkey agar and incubated overnight at $37^{0} \mathrm{C}$ as they are the common media for isolation of organism. The following day the colonies on the media were subjected to Gram staining and motility testing by hanging drop method, following which appropriate biochemical tests were employed. Initially, catalase and coagulase tests were done on all types of colonies to determine the organism. Antimicrobial susceptibility test (AST) was performed for pathogenic bacteria isolated from the specimen. Muller Hinton Agar (MHA) is considered best media for routine AST, hence the test bacteria was inoculated on the solid media (MHA) and filter paper disks impregnated with appropriate concentration of the antibiotic solution were used. Sensitivity to the drug was determined by the zone of inhibition of the bacterial growth around the disk.

\section{Statistical analysis}

Data was entered in Microsoft Excel 2010 and analyzed using Statistical Package for Social sciences version 21. Descriptive statistics were used and results were expressed as frequency. Cases were categorized into children less than 15 years and those who were $\geq 15$ years; geriatrics compared to others and also as per the site of bite. Binary logistic regression was applied on the above mentioned categories to find the strength of association of sensitivity of various antibiotics in different scenarios. Ethical Committee approval was obtained prior to pursuance of the research. 


\section{Results}

We obtained a total of 123 such cases during the study period, out of which $28(22.76 \%)$ cases had consumed different antibiotics 72 hours prior to reporting to the ARC center of the institution. Swabs could not be taken from $13(10.56 \%)$ cases of superficial injuries, infants were $4(3.25 \%)$ and $3(2.43 \%)$ of them did not give consent to take swabs from the wound and hence we were left with 75 cases for antibiotic sensitivity. Out of the 123 cases registered for the study, 96 cases were referred from different health centres. Of these 96 referral cases, we obtained cephalosporins (cefixime and cefpodoxime)were prescribed to most 43(44.79\%), followed by floroquinolones (ofloxacin, ciprofloxacin) 23(23.95\%), linezolid $17(17.7 \%)$ and amoxicillin clavulanic acid $13(13.54 \%)$.

A total of 75 swabs were finally subjected to a battery of microbiological tests and swab culture. Table 1 summarizes the patient characteristics along with the factors associated with animal exposure. Geriatrics ( $\geq 60$ years) accounted for maximum (46.7\%) of the cases with deep wounds among the sample followed by an almost equal distribution among patients aged 16-60 years (29.3\%) and patients aged 1-15 years 18 (24\%). Males and females shared an equal chance of exposure to severe degree of animal bite i.e $50.7 \%$ and $49.3 \%$ respectively. Dogs were a major (78.7\%) cause of injury affecting lower limbs and abdomen in $52 \%$, upper limb in $30.7 \%$ and Head and neck in $17.3 \%$ of individuals. Majority (66.7\%) people were from the area which the tertiary care Hospital caters. People reporting to the ARC within 0-3 days was as high as $97.3 \%$ among the selected cases. Study subjects $(58.7 \%)$ reported of being bitten by suspected rabid animal. Majority (60\%) had unprovoked bite from stray animal $(88 \%)$ and $65.3 \%$ were traceable.

Figure 1 depicts the various bacteria found in the culture from the wound. Bacterial growth was absent in $18.7 \%$ of specimen. However, Staphylococcus accounted for $52 \%$ in the cultures from the wounds followed by Pseudomonas $(19.2 \%)$ and Acinetobacter (16.2\%) and Kleibsiella were the least (14.7\%).

Drug sensitivity to various antibiotics were checked for by culture. Drug used for sensitivity testing were Cotrimoxazole, Gentamycin, Linezolid, Doxycyclin, Clindamycin, Ciprofloxacin, Piperacillin, Amikacin, Imipenam, Amoxycillin + Clavulanic acid, Cefepime, Tobramycin, Ceftazidime and Meropenam. Out of all the drugs tested, maximum sensitivity was found for Linezolid (80\%) followed by Cefepime (69.2\%) Amoxycillin + clavulanic acid (60\%), Tobramycin (50\%), Cotrimoxazole (48.7\%) and Ciprofloxacin (44.2\%). Maximum resistance was found for Piperacillin (87.8\%) and least for Linezolid (20\%) and Cefepime (30.7\%).

Cases were categorized into children less than 15 years, geriatrics ( $\geq 60$ years), wounds on lower limb and abdomen and wounds on face to find the antibiotic sensitivity pattern. We found Cotrimoxazole, Doxyclicline, Ciprofloxacin and Meropenamto have significantly high sensitivity among children less than 15 years. Cotrimoxazole and Ciprofloxacin were found to be 3.7 times and 3.4 times sensitive in children as compared to adults. Among geriatrics, Linezolid, Doxycycline, Tobramycin and Imipenem was found to have significantly high sensitivity to wounds caused by animal bites in geriatrics. Linezolid was found to be 3.11 times more sensitive in geriatrics as compared to adults. Amoxycillin and Piperacillin were found to have significant difference in sensitivity for wounds of lower limbs and abdomen as compared to wounds of other sites. Likewise, Gentamicin, Linezolid, Piperacillin and Imipenem were found to be sensitive to facial injuries as compared to injuries at other sites. 
Table 1: Factors related to animal exposure among the study participants $(\mathrm{N}=75)$

\begin{tabular}{|l|c|c|c|c|}
\hline Sl no & Characteristics & & Number & Percentage (\%) \\
\hline 1 & Age (in years) & $1-15$ years & 18 & 24 \\
& & $16-59$ years & 22 & 29.3 \\
& & $\geq 60$ years & 35 & 46.7 \\
\hline 2 & Gender & Male & 38 & 50.7 \\
& & Female & 37 & 49.3 \\
\hline 3 & Site of injury & Upper limb & 23 & 30.7 \\
& & Abdomen and lower limb & 39 & 52.0 \\
& & Head and neck & 13 & 17.3 \\
\hline 4 & Cause of injury & Dog & 59 & 78.7 \\
& & Others & 16 & 21.3 \\
\hline 5 & District of Residence & Within same district* & 50 & 66.7 \\
& & Outside district & 25 & 33.3 \\
\hline 6 & Days passed since bite & $0-3$ days & 73 & 97.3 \\
& & $>3$ days & 2 & 2.7 \\
\hline 7 & Suspected Rabid Animal & Yes & 44 & 58.7 \\
& & No & 31 & 41.3 \\
\hline 8 & Type of Injury & Provoked & 30 & 40 \\
& & Unprovoked & 45 & 60 \\
\hline 9 & Nature of Animal & Stray & 66 & 88 \\
& & Pet & 9 & 12 \\
\hline 10 & Traceable & Yes & 49 & 65.3 \\
& & No & 26 & 34.7 \\
\hline 11 & Received Primary Treatment & Yes & 44 & 58.7 \\
& & No & 31 & 41.3 \\
\hline
\end{tabular}

*Cases residing in the same district as of the tertiary care teaching hospital.

Table 2: Association of various factors with different antibiotic sensitivity. ( $\mathrm{N}=75)$

\begin{tabular}{|c|c|c|c|c|c|c|}
\hline $\begin{array}{l}\text { SI } \\
\text { no }\end{array}$ & Drug & Characteristics & Sensitive & $\begin{array}{l}\text { Resistant/no } \\
\text { growth }\end{array}$ & $\begin{array}{c}\mathbf{P} \\
\text { value }\end{array}$ & $\begin{array}{c}\text { Odds ratio } \\
\text { Confidence Interval }\end{array}$ \\
\hline 1 & Cotrimoxazole & \multirow{4}{*}{$\begin{array}{c}\text { Age }<15 \text { years } \\
\geq 15 \text { years }\end{array}$} & $\begin{array}{c}9(50) \\
12(21.1)\end{array}$ & $\begin{array}{c}9(50) \\
45(78.9)\end{array}$ & 0.01 & $3.7(1.22-11.51)$ \\
\hline 2 & Doxycycline & & $\begin{array}{c}0(0) \\
20(35.1)\end{array}$ & $\begin{array}{l}18(100) \\
37(64.9)\end{array}$ & 0.003 & $1.54(1.27-1.86)$ \\
\hline 3 & Ciprofloxacin & & $\begin{array}{l}11(61.1) \\
18(31.6)\end{array}$ & $\begin{array}{r}7(38.9) \\
39(68.4)\end{array}$ & 0.02 & $3.40(1.13-10.22)$ \\
\hline 4 & Meropenam & & $\begin{array}{c}0(0) \\
11(19.3)\end{array}$ & $\begin{array}{l}18(100) \\
46(80.7)\end{array}$ & 0.04 & $1.23(1.09-1.40)$ \\
\hline 5 & Doxycycline & \multirow[t]{4}{*}{$\begin{array}{l}\text { Age } \geq 60 \text { years } \\
<60 \text { years }\end{array}$} & $\begin{array}{l}14(40) \\
6(15)\end{array}$ & $\begin{array}{l}21^{\prime}(60) \\
34(85)\end{array}$ & 0.01 & $3.77(1.25-11.35)$ \\
\hline 6 & Linezolid & & $\begin{array}{c}20(57.1) \\
12(30)\end{array}$ & $\begin{array}{c}15(42.9) \\
28(70)\end{array}$ & 0.01 & $3.11(1.20-8.05)$ \\
\hline 7 & Imipenam & & $\begin{array}{c}17(48.6) \\
8(20)\end{array}$ & $\begin{array}{l}18(51.4) \\
32(80)\end{array}$ & 0.009 & $3.77(1.36-10.47)$ \\
\hline 8 & Tobramycin & & $\begin{array}{c}0(0) \\
5(12.5)\end{array}$ & $\begin{array}{l}35(100) \\
35(87.5)\end{array}$ & 0.03 & $1.14(1.01-1.28)$ \\
\hline 9 & Piperacillin & \multirow[t]{2}{*}{$\begin{array}{l}\text { †LL \&Abd } \\
\text { Others }\end{array}$} & $\begin{array}{l}21(53.8) \\
8(22.2)\end{array}$ & $\begin{array}{l}18(46.2) \\
28(77.8)\end{array}$ & 0.005 & $4.08(1.49-11.17)$ \\
\hline 10 & $\begin{aligned} & \text { Amoxycillin } \\
&+ \text { clavulanic acid }\end{aligned}$ & & $\begin{array}{c}0(0) \\
4(11.1)\end{array}$ & $\begin{array}{l}39(100) \\
32(88.9)\end{array}$ & 0.03 & $1.12(1.00-1.26)$ \\
\hline 11 & Gentamicin & \multirow[t]{4}{*}{$\begin{array}{l}\text { Face } \\
\text { Others }\end{array}$} & $\begin{array}{l}13(100) \\
24(38.7)\end{array}$ & $\begin{array}{c}0(0) \\
38(61.3)\end{array}$ & 0.00 & $2.58(1.88-3.53)$ \\
\hline 12 & Linezolid & & $\begin{array}{l}11(84.6) \\
24(38.7)\end{array}$ & $\begin{array}{c}2(15.4) \\
38(61.3)\end{array}$ & 0.003 & $8.70(1.77-42.74)$ \\
\hline 13 & Piperacillin & & $\begin{array}{c}0(0) \\
29(46.8)\end{array}$ & $\begin{array}{l}13(100) \\
33(53.2)\end{array}$ & 0.002 & $1.87(1.48-2.37)$ \\
\hline 14 & Imipenam & & $\begin{array}{c}0(0) \\
25(40.3)\end{array}$ & $\begin{array}{l}13(100) \\
37(59.7)\end{array}$ & 0.005 & $1.67(1.36-2.05)$ \\
\hline
\end{tabular}




\section{JMSCR VoI||08||Issue||01||Page 380-387||January}

Fig 1: Causative bacteria present in the wound

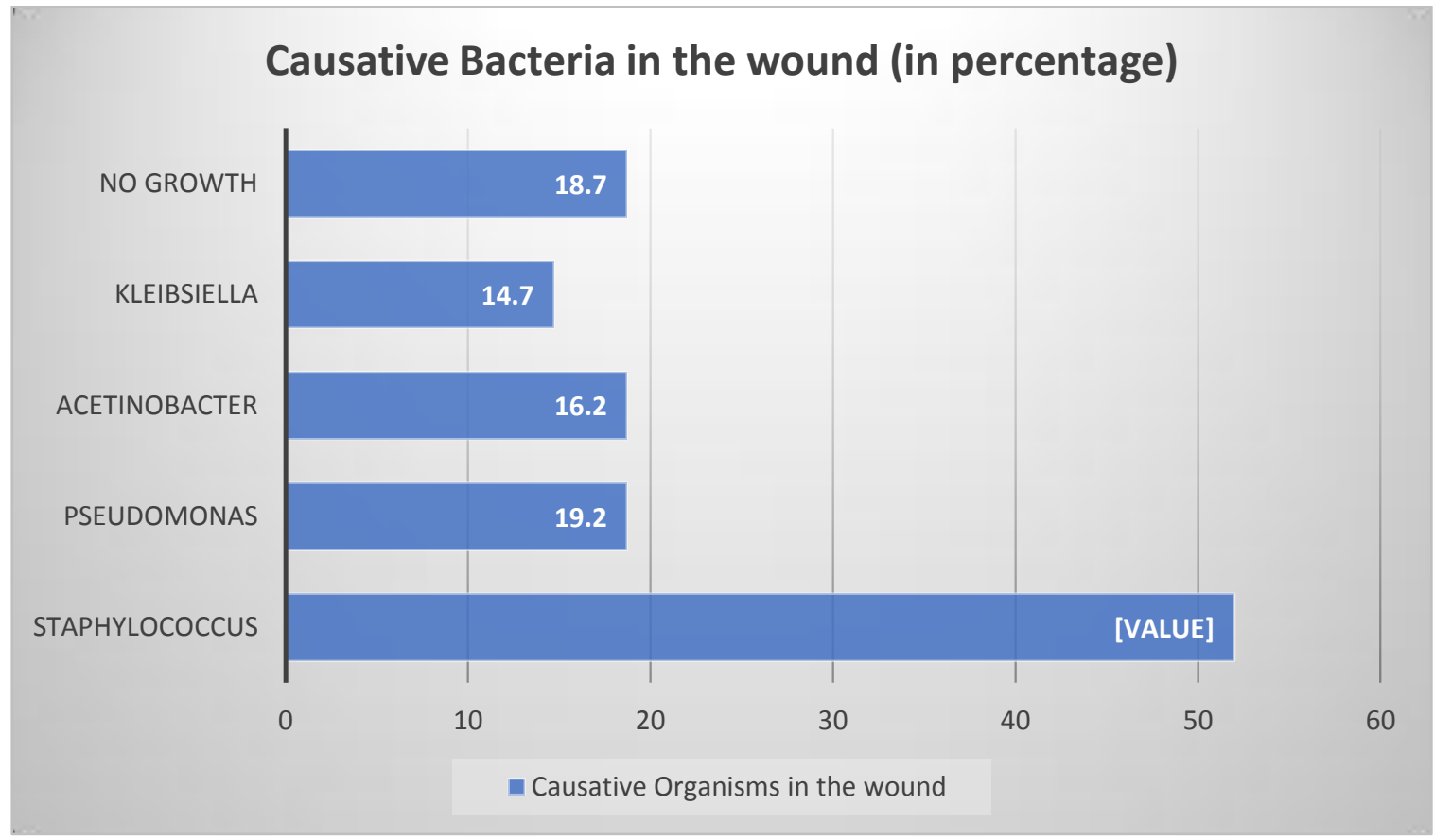

Figure 2: Drug sensitivity of the bacteria found in the wounds to various antibiotics

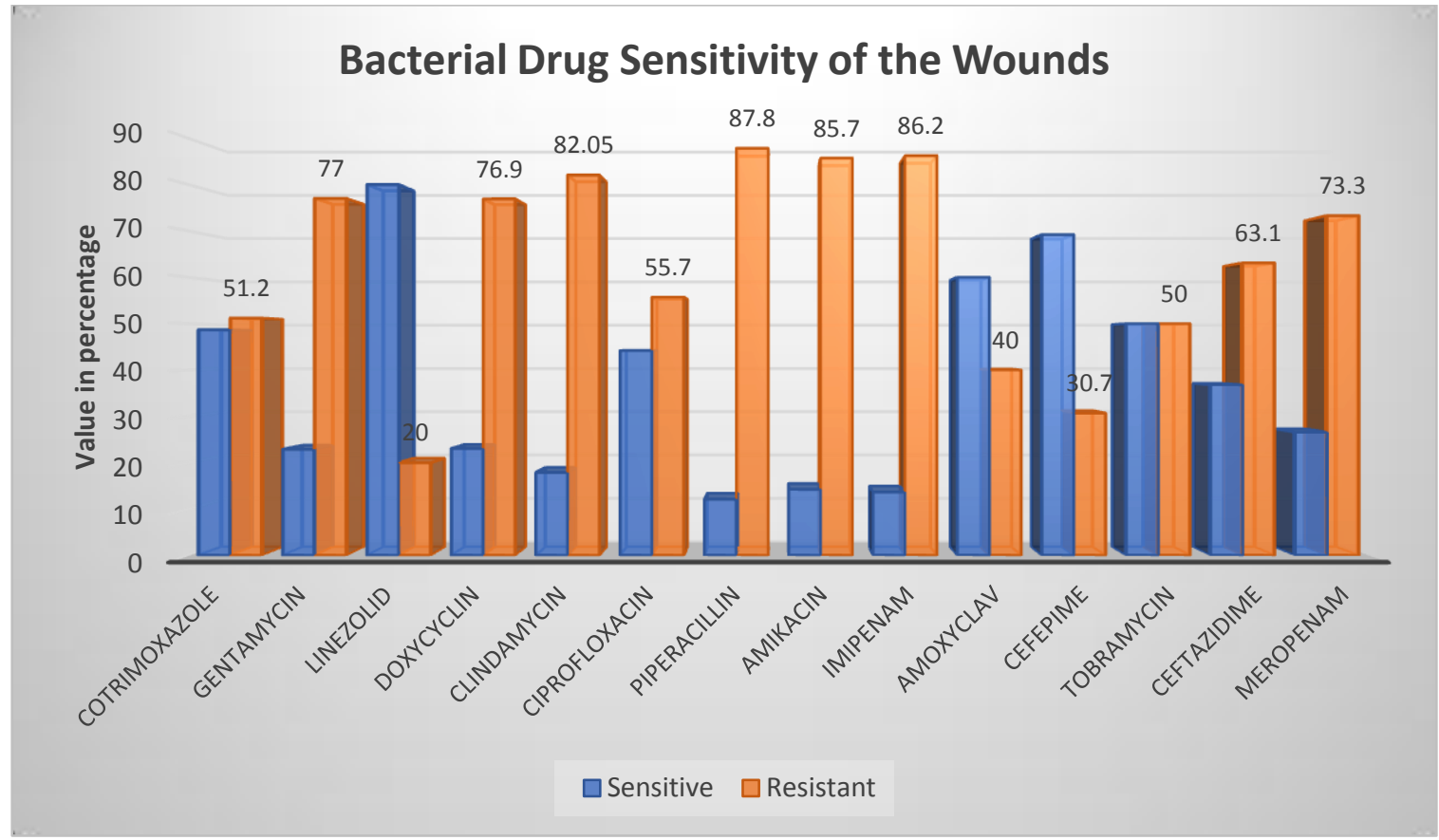

\section{Discussion}

Geriatrics formed nearly half of the cases with severe deep/lacerated wound. This brings out the very fact that although children form a major chunk of ARC OPD attendance but geriatrics fall prey to more severe bites. This could be mainly because of frailty and less defensiveness on part of elderly. Mohanty S et $\mathrm{al}^{3}$ and Mahendra B J et $\mathrm{al}^{4}$ found children to be mostly affected as compared to adults but we could not substantiate for severity of wounds in any of the age group. The study highlights an equal exposure to severe degree of animal bites among both males and females. However, there is paucity of evidence as per severity of wounds due to animal bites in either gender, although males being more affected than female is found in many other studies. ${ }^{3,4}$ Lower limbs and abdomen were the main site of injury 
which was similar to the findings of Mohanty $\mathrm{S}$ et $\mathrm{al}^{5}$ and Jesha Mohammedali. $M$ et $\mathrm{al}^{6}$.Easier accessibility of these sites to the animals might be the reason of more attacks to lower limbs and abdomen. Dogs have been the major vector in spreading the deadly disease Rabies and nearly 8 out of 10 cases have been bitten by dogs in our study, as also found by Mohua Biswas et al. ${ }^{2}$ Due to easier accessibility and availability of biologicals against animal bite at this tertiary care hospital, every seventh case belonged to the same district. A significant finding of our study was the fear marked amongst the study subjects regarding the deadliness of Rabies and thereby leading to $97.3 \%$ of subjects to report within 0-3 days. Nearly 6 out of 10 cases suspected of being bitten by rabid animal. Stray animals accounted for nearly $90 \%$ of bites of which maximum $(60 \%)$ were unprovoked bites.

Bacteria found inhabiting on the injuries afflicted by animals were mainly Staphylococcus, Pseudomonas, Acenitobacter and Klebsiella. Staphylococcus accounted for half of the cases as was also supported by previous studies conducted by Sibani Barman et $\mathrm{al}^{7}$ and Mohuabis was et $\mathrm{al}^{2}$. In a study conducted by Talan D. A et $\mathrm{al}^{8}$ Pasteurellacanis was the most common species isolated from infected dog bite wounds .Results of antibiotic susceptibility and resistance was an alarming note to the rampant usage of antibiotics and its decreasing efficacy before attending a tertiary care hospital. This hints to the easy use, accessibility and irrational use at the peripheral health institutions where the patients first come in contact, thereby causing a havoc of mass drug resistance to mankind in future. In this era of microbial resistance to antibiotics and a call from WHO on antibiotic susceptibility, this study would definitely be another eye-opener towards the irrational use of antibiotics. Maximum sensitivity was marked among Linezolid (80\%) and Amoxycillin + Clavulinic Acid (60\%). This was similar to the study conducted by Mohua Biswas et $\mathrm{al}^{2}$ where the maximum sensitivity was found to Linezolid (100\%) and Amoxycillin clavulanic acid (70\%). This also hints to the precious antibiotics left with us and awakening call for their proper use. Staphylococcus being the most common organism, and with the development of MRSA and VRSA, Linezolid with a sensitivity of as high as $80 \%$ as found in our study stands out to be one of the best choices for management of the deep wounds caused by animal bite. Linezolid is useful in the treatment of resistant gram positive cocci (aerobic and anaerobic) and bacillary infections ${ }^{9}$. It is specifically advised for uncomplicated and complicated skin and soft tissue infections. However, in order to preserve this valuable drug for resistant cases this must be kept as a reserve drug and not used rampantly. A thorough evaluation of the referred prescriptions revealed Cefiximeas first antibiotic advised in majority of cases which shows the irrational use of antibiotics as Cefixime is not active on the main culprit Staphylococcus. Pseudomonas was found in onefifth of cases and among Cephalosporin, Cefepime, the fourth generation cephalosporin which has sensitivity in nearly $70 \%$ of cases and has stronger activity on both Pseudomanas and Staphylococcus is a very good alternative for severe wounds leading to septicemia in immunocompromised patients. Out of all the commonly used antibiotics for wound management, amoxicillin with clavulanic acid with a sensitivity of $60 \%$ is a better choice for wound management in case of animal bite. Amoxicillin with clavulanic acid acts against resistant Staph aureus and Klebsiella, hence it should be advised for management of such cases considering both benefit and resistance.

Cotrimoxazole is a fixed dose combination (FDC) of trimethoprim and sulfamethoxazole which are bacteriostatic individually but in a FDC form it is bacteriocidal against many organisms. It has a wide spectrum of antibacterial action, against sulphonamide resistant Staph aureus, Klebsiella, E.coli, Enterobacter etc. We found cotrimoxazole to be nearly 4 times significantly highly sensitive in children less than 15 years. Similarly among 
oral antibiotics, Ciprofloxacin was found to be highly sensitive with an odds of 3.4 among children less than 15 years. However, keeping in view the present scenario of resistance to antimicrobials, Cotimoxazole with a sensitivity of nearly $50 \%$ would be a better option for treating children with animal bites.

Similarly, the drug sensitivity pattern when observed among the geriatrics was found to be a little different than the children. Oral antibiotics such as Linezolid and Doxycycline were found to have very high significant sensitivity among the geriatrics. Linezolid was found to be effective in 8 out of 10 cases with an odds of 3.1 and a confidence interval ranging from 1.20 to 8.05. Hence, rational use of drugs with first line drugs along with the knowledge of sensitivity pattern of antibiotics will be a step forward in deterring antibiotic resistance.

Cases were also analyzed for antibiotic sensitivity pattern as per the site of bite. Cases with bites mainly on lower limbs and abdomen were found to have a significant higher sensitivity for Amoxycillin + clavulanic acid. Likewise cases with bites on face were found to have 8 times sensitive to Linezolid. Staphylococcus being a major contributor, drugs such as Linezolid and Amoxycillin have rightfully been sensitive and hence its use is a must in animal bite cases.

\section{Conclusion}

Geriatrics were found to be at a higher risk for severe animal bites. Drugs such as Amoxycilllin with clavulanic acid and Linezolid should be used judiciously in animal bite cases as they were found to have higher sensitivity. First line antibiotics should be more in use than using reserve drugs or injectable. This was supported by the fact that resistance was mainly seen among the injectable drugs which are rampantly used in the peripheral set-up.

\section{Acknowledgment}

We highly appreciate the cooperation of the patients as well as the immense help from the
Department of Microbiology in conducting this study.

\section{Sources of support- Nil}

\section{References}

1. Gardiner Harris. Where Streets are thronged with strays baring fangs. Asia Pacific, New Delhi Journal. 6 August 2012. Available on http://www.nytimes.com/2012/08/07/worl d/asia/India-stray-dogs-are-amenace.html?pagewanted $=$ all\&_r=0. Last accesed on $10^{\text {th }}$ October, 2019.

2. Mohua Biswas1, Durga Madhab Satapathy. Bacterial contamination and antibiotic sensitivity pattern of animal bite wounds: a study in the anti-rabies clinic of scb medical college, cuttack, odisha. APCRI Journal, Volume XX Issue I July 2018, p 11-13.

3. Mohanty S, Agasti N, Behera TR, Das S. Profile of re-exposure animal bite cases at anti-rabies clinic of MKCG Medical College, Berhampur, Odisha. APCRI Journal July (2017), Vol (XIX), Issue 1, 24-27.

4. Mahendra BJ, Harish BR, Vinay M. A study of factors influencing compliance to IDRV at Anti-Rabies Clinic of Mandya Institute of Medical Sciences, Mandya. APCRI Journal, Vol XI, Issue I, Jul 2009.

5. Smaraki Mohanty, Tapas Ranjan Behera, Nupur Patnaik. Clinical Safety of an equine rabies immunoglobulin. APCRI Journal Volume XIX, Issue II, Jan 2018, p 9-12.

6. Jesha Mohammedali. M, Jo Martin, Thomas Bina, Lucy Raphel, Lailabi, Jaydev. Compliance to IDRV at the Anti Rabies Clinic in a tertiary Care hospital in Northern Kerela. APCRI Journal Volume XII, Issue II, jan 2011, p 21-24.

7. Sibani Barman, E. Preetha, P. Swarnalatha, KN Venkataramana B. Sekar. 
Identification of infectious organisms from animal wounds and characterization of toxin by invitro and in vivo methods. APCRI Journal vol XIV, Issue I,july 2012, p 25-29.

8. Talan D. A., Citron D. M., Abrahamian F. M., Moran G. J., Goldstein E. J. C. 1999.Bacteriologic analysis of infected dog and cat bites. N. Engl. J. Med. 340:8592 [PubMed]

9. K D Tripathi, Essentials of medical pharmacology. Seventh edition. Jaypee publishers. 